\title{
Different inflammatory phenotypes in adults and children with acute asthma
}

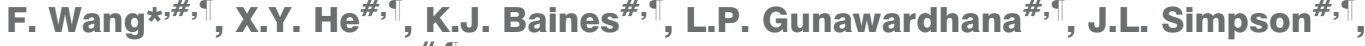 \\ F. Li* and P.G. Gibson*,\#,
}

ABSTRACT: Inflammatory phenotypes are recognised in stable adult asthma, but are less well established in childhood and acute asthma. Additionally, Chlamydophila pneumoniae infection as a cause of noneosinophilic asthma is controversial. This study examined the prevalence of inflammatory phenotypes and the presence of current $C$. pneumoniae infection in adults and children with stable and acute asthma.

Adults with stable $(n=29)$ or acute $(n=22)$ asthma, healthy adults $(n=11)$, children with stable $(n=49)$ or acute $(n=28)$ asthma, and healthy children $(n=9)$ underwent clinical assessment and sputum induction. Sputum was assessed for inflammatory cells, and DNA was extracted from sputum cell suspensions and supernatants for C. pneumoniae detection using real-time PCR.

The asthma phenotype was predominantly eosinophilic in children with acute asthma (50\%) but neutrophilic in adults with acute asthma (82\%). Paucigranulocytic asthma was the most common phenotype in both adults and children with stable asthma. C. pneumoniae was not detected in $99 \%$ of samples.

The pattern of inflammatory phenotypes differs between adults and children, with eosinophilic inflammation being more prevalent in both acute and stable childhood asthma, and neutrophilic inflammation being the dominant pattern of acute asthma in adults. The aetiology of neutrophilic asthma is unknown and is not explained by the presence of current active C. pneumoniae infection.

KEYWORDS: Acute asthma, adults, asthma, children, Chlamydophila pneumoniae, phenotypes

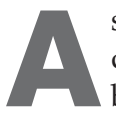
sthma is a chronic inflammatory airway disease characterised by episodic exacerbations. There is heterogeneity in the airway inflammatory response in asthma that can be related to both exposures, such as allergen and infection, and to treatment [1]. We have previously categorised stable adult asthma into four inflammatory phenotypes based on induced sputum inflammatory cell counts [2]. These include eosinophilic, neutrophilic, mixed granulocytic and paucigranulocytic asthma, defined by the presence and/or absence of airway eosinophilis and neutrophils. The occurrence and relative frequency of these phenotypes in acute asthma and childhood asthma are not known. Similarly, the relationship of specific phenotypes to infectious triggers has not been well studied.

While exposure to allergen in a sensitised individual can elicit an eosinophilic response, the triggers of the noneosinophilic phenotypes have been less well studied. Chronic infection is a possible cause, since infections typically elicit a neutrophil response similar to that observed in neutrophilic asthma (NA). This response is mediated via innate immune activation and evidence of innate immune dysregulation in adults with NA has previously been reported [3]. In that study, there was significant upregulation of Toll-like receptor (TLR)2 gene expression and a corresponding increase in CXC ligand 8 (interleukin (IL)-8) in NA. While viral infection is a frequent cause of acute asthma and airway neutrophilia, this is usually mediated by TLR3 or TLR7 activation and does not explain the neutrophilic responses that are seen in stable asthma. These data suggest that other organisms capable of both chronic and acute infection, and eliciting TLR2 mediated responses, may be relevant in noneosinophilic asthma (NEA).

Chlamydophila pneumoniae is a potential cause of NEA, since it causes both an acute infection and a chronic persistent infectious state with reactivation [4]. C. pneumoniae infects lung tissue and elicits an innate immune response that is mediated via TLR2 [5] and results in neutrophilic inflammation [4]. C. pneumoniae has also been reported to worsen asthma severity [6] and be a cause of asthma exacerbation [7]. NA occurs

\section{AFFILIATIONS}

*Dept of Pathogenobiology, Norman Bethune College of Medicine, Jilin University, Changchun, China, ${ }^{\#}$ Centre for Asthma and Respiratory Disease, University of Newcastle, Callaghan, NSW, and

"Dept of Respiratory and Sleep Medicine, Hunter Medical Research Institute, John Hunter Hospital, New Lambton, NSW, Australia.

CORRESPONDENCE

P.G. Gibson

Dept of Respiratory Medicine John Hunter Hospital

Locked Bag 1

Hunter Region Mail Centre

Newcastle

NSW 2310

Australia

E-mail: Peter.gibson@

hnehealth.nsw.gov.au

Received:

Nov 022010

Accepted after revision:

Dec 162010

First published online:

Jan 132011 
typically in older adults and there is evidence that $C$. pneumoniae infection might precede the onset of adult asthma, as well as persisting in stable chronic asthmatics [8]. Furthermore, the noneosinophilic phenotype of severe asthma is improved by macrolide antibiotics, a class of drugs that are active against C. pneumoniae [9]. C. pneumoniae detection may be higher in children or in acute exacerbations of asthma, where there is also heterogeneity of the inflammatory response. Thus, C. pneumoniae may play a potential role in modulating the different inflammatory phenotypes of asthma, and NA in particular. The potential mechanisms include chronic persistence of C. pneumoniae in NEA, or acute infection with clearance of the organism but persistent immune reprogramming that leads to the immunopathology of NEA $[4,10]$. The latter mechanism is biologically plausible and has support from model systems of asthma [4, 10]. Real-time PCR is an effective method for detecting $C$. pneumoniae using induced sputum [11]. The major outer membrane protein (MOMP) gene has previously been shown to be a good genetic marker for $C$. pneumoniae in other real-time PCR-based studies [12, 13].

The aim of this study was to examine the differences in inflammatory phenotype of asthma among adults with stable asthma (ASAs), adults with acute asthma (AAAs), children with stable asthma (CSAs) and children with acute asthma (CAAs), and to investigate the role of chronic C. pneumoniae infection in the NEA phenotypes. We hypothesised that there would be differences in inflammatory phenotypes between adults and children with asthma, specifically that acute asthma in children and adults would be similar and exhibit a neutrophilic phenotype, whereas in stable asthma the eosinophilic asthma (EA) phenotype would be more prevalent in children compared with adults. We also hypothesised that $C$. pneumoniae infection may be common with NEA phenotypes. To assess these hypotheses, we selected subjects who had participated in previous studies [3, 14, 15] and evaluated inflammatory phenotypes from induced sputum cell counts and C. pneumoniae using DNA extracted from sputum samples.

\section{MATERIALS AND METHODS}

\section{Participants}

There were four groups of asthmatics and two groups of healthy controls selected for study. Nonsmoking ASAs $(n=29)$ and AAAs $(n=22)$ had asthma defined using the American Thoracic Society criteria, and each had a doctor's diagnosis of symptomatic asthma. CSAs $(n=49)$ and CAAs $(n=28)$ had asthma diagnosed by a respiratory paediatrician based on clinical and lung function criteria. The subjects with acute asthma were recruited soon after presentation to the John Hunter Hospital emergency department (New Lambton, NSW, Australia) with an acute exacerbation of asthma, as described previously [3, 14, 15]. Participants were recruited within $4 \mathrm{~h}$ of attendance and they were all pre-treated with an inhaled $\beta_{2^{-}}$ agonist before sputum induction. The stable subjects had no recent (past 4 weeks) respiratory infection, asthma exacerbation or medication change. Two comparison groups were selected comprising nonsmoking healthy adults $(n=11)$ and healthy children $(n=9)$ with no respiratory symptoms and normal lung function. The subjects were selected from the laboratory database based on the selection criteria above, and the availability of induced sputum cell counts and stored sputum samples for analysis. Some of these subjects were enrolled in previous studies [3, 14]. All participants gave informed consent prior to their inclusion in the study and Hunter New England Area Health Service Human Research Ethics Committees (New Lambton, NSW) approved this study.

\section{Sputum induction and analysis}

Spirometry (KoKo PD Instrumentation, Louisville, CO, USA) and sputum induction were performed using hypertonic saline ( $4.5 \%$ for stable asthma and controls; $0.9 \%$ for acute asthma) delivered via an ultrasonic nebuliser, as previously described [16]. The induced sputum was collected, and mucocellular portions were selected, separated from the whole sputum sample and dispersed using dithiothreitol [17]. The suspension was then filtered and a total cell count was obtained using trypan blue exclusion. The remaining sample was centrifuged and the cell supernatants were stored at $-80^{\circ} \mathrm{C}$. The cell pellet was diluted with PBS to $1 \times 10^{6}$ cells per mL. Cytospins were prepared, stained with May-Grunwald Giemsa and a differential cell count was obtained from 400 nonsquamous cells. The remaining cell suspension was stored at $-80^{\circ} \mathrm{C}$ in PBS.

\section{Classification of asthma phenotypes}

Based on previous studies $[2,16]$, subjects with $>3 \%$ sputum eosinophils were classified as EA and those with $>61 \%$ sputum neutrophils and $<3 \%$ eosinophils were classified as NA. Subjects with $>61 \%$ sputum neutrophils and sputum $>3 \%$ eosinophils were classified as mixed granulocytic asthma, and those with $<61 \%$ sputum neutrophils and $<3 \%$ eosinophils were classified as paucigranulocytic asthma.

\section{C. pneumoniae detection}

Genomic DNA was isolated from cell suspensions and cell supernatants of induced sputum using the QIAamp DNA Mini Kit (Qiagen Inc., Valencia, CA, USA), according to the manufacturer's instructions for isolation of bacterial DNA. DNA was eluted in $100 \mu \mathrm{L}$ Buffer $\mathrm{AE}$ and stored at $-20^{\circ} \mathrm{C}$. Quality and quantity of extracted DNA was assessed using a NanoDrop 2000 (Thermo Fisher Scientific Inc., Wilmington, DE, USA). Real-time PCR was performed with a 7500 Real Time PCR system (Applied Biosystems, Foster City, CA, USA). C. pneumoniae was detected using the Quantification of Chlamydophila pneumoniae Major Outer Membrane Protein Gene Advanced kit (PrimerDesign ${ }^{\mathrm{TM}}$ Ltd, Southampton, UK), according to the manufacturer's instructions. The pathogenspecific primer/probe mix (carboxyfluorescein labelled), pathogen positive control template and endogenous $\beta$-actin $(A C T B)$ primer/probe mix inside the kit were used to detect $C$. pneumoniae MOMP gene and endogenous ACTB gene.

\section{Validation of C. pneumoniae detection in sputum}

Suitability of sputum processing and DNA extraction methods for recovery of C. pneumoniae DNA were assessed in a separate experiment (see Results section). Briefly, an induced sputum sample was divided into several aliquots $(200 \mu \mathrm{L})$ and each aliquot was combined with different amounts of $C$. pneumoniaepositive DNA (PrimerDesign ${ }^{\mathrm{TM}} \mathrm{Ltd}$ ). Then, aliquots were processed, and cell suspensions and supernatants were obtained. The processed samples were stored for 1 day at $-80^{\circ} \mathrm{C}$ to emulate laboratory sample storage conditions before 
TABLE 1 Subject characteristics by disease group for adults and children

\begin{tabular}{|c|c|c|c|c|c|c|}
\hline & \multicolumn{3}{|c|}{ Adults } & \multicolumn{3}{|c|}{ Children } \\
\hline & Controls & Stable asthma & Acute asthma & Controls & Stable asthma & Acute asthma \\
\hline Subjects & 11 & 29 & 22 & 9 & 49 & 28 \\
\hline Age $^{\#}$ yrs & $59(41-74)$ & $56(51-65)$ & $43(30-53)$ & $10(9-11)$ & $11(9-13)$ & $12(8-17)$ \\
\hline Males/females & $6 / 5$ & $12 / 17$ & $7 / 15$ & $1 / 8$ & $32 / 17^{\star \star *}$ & $15 / 13$ \\
\hline ICS use & $0(0)$ & $26(90)^{* \star *}$ & $18(82)^{* \star *}$ & $0(0)$ & $30(73)^{\star \star *}$ & $19(68)^{\star \star \star}$ \\
\hline $\begin{array}{l}\text { BDP-equivalent ICS } \\
\text { dose }^{*} \mu \mathrm{g} \cdot \text { day }^{-1}\end{array}$ & 0 & $1537 \pm 958$ & $1417 \pm 787$ & 0 & $484 \pm 325$ & NA \\
\hline $\mathrm{FEV}_{1}{ }^{\#} \%$ pred & $100.8(91.9-108.4)$ & $71.4^{+}(59.5-87.2)$ & $73.0^{+}(50.0-77.0)$ & $92(86-103)$ & $91(85-105)$ & $53^{+}(41-69)$ \\
\hline FEV $_{1} /$ FVC $^{\#} \%$ & $81(71-88)$ & $65^{+}(58-74)$ & $74(65-80)$ & $91(85-97)$ & $80(77-85)$ & NA \\
\hline
\end{tabular}

Data are presented as $\mathrm{n}$, median (interquartile range), $\mathrm{n}(\%)$ or mean \pm SD. Fisher's exact or the Chi-squared test was used, unless otherwise stated. ICS: inhaled corticosteroid; BDP: beclomethasone dipropionate; FEV1: forced expiratory volume in $1 \mathrm{~s}$; \% pred: \% predicted; FVC: forced vital capacity; NA: not assessed. ${ }^{\#}$ : KruskalWallis test; ${ }^{\bullet}$ : unpaired t-test; ${ }^{+}: p<0.008$ versus healthy controls in post hoc test. ${ }^{* \star *}: p<0.001$ versus healthy controls in post hoc test.

DNA extractions were performed. The DNA were quantified and subjected to quantitative PCR under identical conditions.

\section{Statistical analysis}

All the data were analysed using STATA 9 (StataCorp, College Station, TX, USA). Results were reported as median and interquartile range (IQR), and the two-sample Wilcoxon rank sum test or Kruskal-Wallis test was used to analyse the data. Fisher's exact test or the Chi-squared test were used to analyse categorical data. The Wilcoxon sign rank test was used to analyse paired data, such as DNA concentration both in sputum cell suspensions and sputum cell supernatants. Significance was accepted when $\mathrm{p}$-values were $<0.05$. Post hoc analysis significance was accepted at p-values $<0.017$.

TABLE 2 Induced sputum cell counts by disease group for adults and children

\begin{tabular}{|c|c|c|c|c|c|c|c|c|}
\hline & \multicolumn{4}{|c|}{ Adults } & \multicolumn{4}{|c|}{ Children } \\
\hline & Controls & Stable asthma & Acute asthma & p-value & Controls & Stable asthma & Acute asthma & p-value \\
\hline Subjects $n$ & 11 & 29 & 22 & & 9 & 49 & 28 & \\
\hline $\begin{array}{l}\text { TCC } \times 10^{6} \text { cells } \\
\text { per } \mathrm{mL}\end{array}$ & $4.9(3.2-6.6)$ & $8.0(4.2-10.6)$ & $9.4(3.0-24.7)$ & 0.164 & $3.2(2.1-4.4)$ & $3.2(1.8-7.0)$ & $5.4(2.6-20.9)$ & 0.064 \\
\hline Eosinophils \% & $0.25(0-0.75)$ & $1.00(0.30-2.50)$ & $0.64(0-2.00)$ & 0.056 & $0.50^{\#}(0.25-0.75)$ & $1.75^{\#}(0.50-4.50)$ & $11.75(5.00-21.38)$ & $<0.001$ \\
\hline $\begin{array}{l}\text { Eosinophils } \times 10^{4} \\
\text { cells per } \mathrm{mL}\end{array}$ & $1.35(0-3.29)$ & $6.84(2.43-21.87)$ & $6.02(0-18.11)$ & 0.054 & $1.5^{\#}(0.8-1.8)$ & $5.5^{\#}(1.0-17.7)$ & $56.1(21.8-356.0)$ & $<0.001$ \\
\hline Macrophages \% & $64.3^{\#}(33.0-69.8)$ & $38.3^{\#}(23.3-49.5)$ & $15.0(11.8-27.3)$ & $<0.001$ & $81.3^{\# .9}(75.3-88.0)$ & $58.5^{\#}(36.0-74.0)$ & $18.1(10.5-37.4)$ & $<0.001$ \\
\hline $\begin{array}{l}\text { Macrophages } \times 10^{4} \\
\text { cells per } \mathrm{mL}\end{array}$ & $228(134-314)$ & $207(147-357)$ & $143(86-251)$ & 0.099 & $193(174-373)$ & $166(100-272)$ & $126(63-302)$ & 0.436 \\
\hline $\begin{array}{l}\text { Columnar epithelial } \\
\text { cells } \%\end{array}$ & $1.25(0.25-2.00)$ & $2.50(0.25-5.25)$ & $0.83(0.26-2.76)$ & 0.266 & $0.75(0-2.75)$ & $2.00(0.31-4.25)$ & $3.0(0.38-4.75)$ & 0.374 \\
\hline $\begin{array}{l}\text { Columnar epithelial } \\
\text { cells } \times 10^{4} \text { per } \mathrm{mL}\end{array}$ & $6.84(1.94-13.95)$ & $11.68(2.66-34.02)$ & $5.02(0.76-14.93)$ & 0.185 & $2.61(0-4.95)$ & $5.72(2.18-12.02)$ & $14.33(3.13-30)$ & 0.038 \\
\hline
\end{tabular}

Data are presented as median (interquartile range), unless otherwise stated. The Kruskal-Wallis test was used. Bold indicates statistically significant p-values. TCC: total cell count. ${ }^{\#}: p<0.008$ compared with acute asthma in post hoc test; ${ }^{\circ}: p<0.008$ compared with stable asthma in post hoc test. 

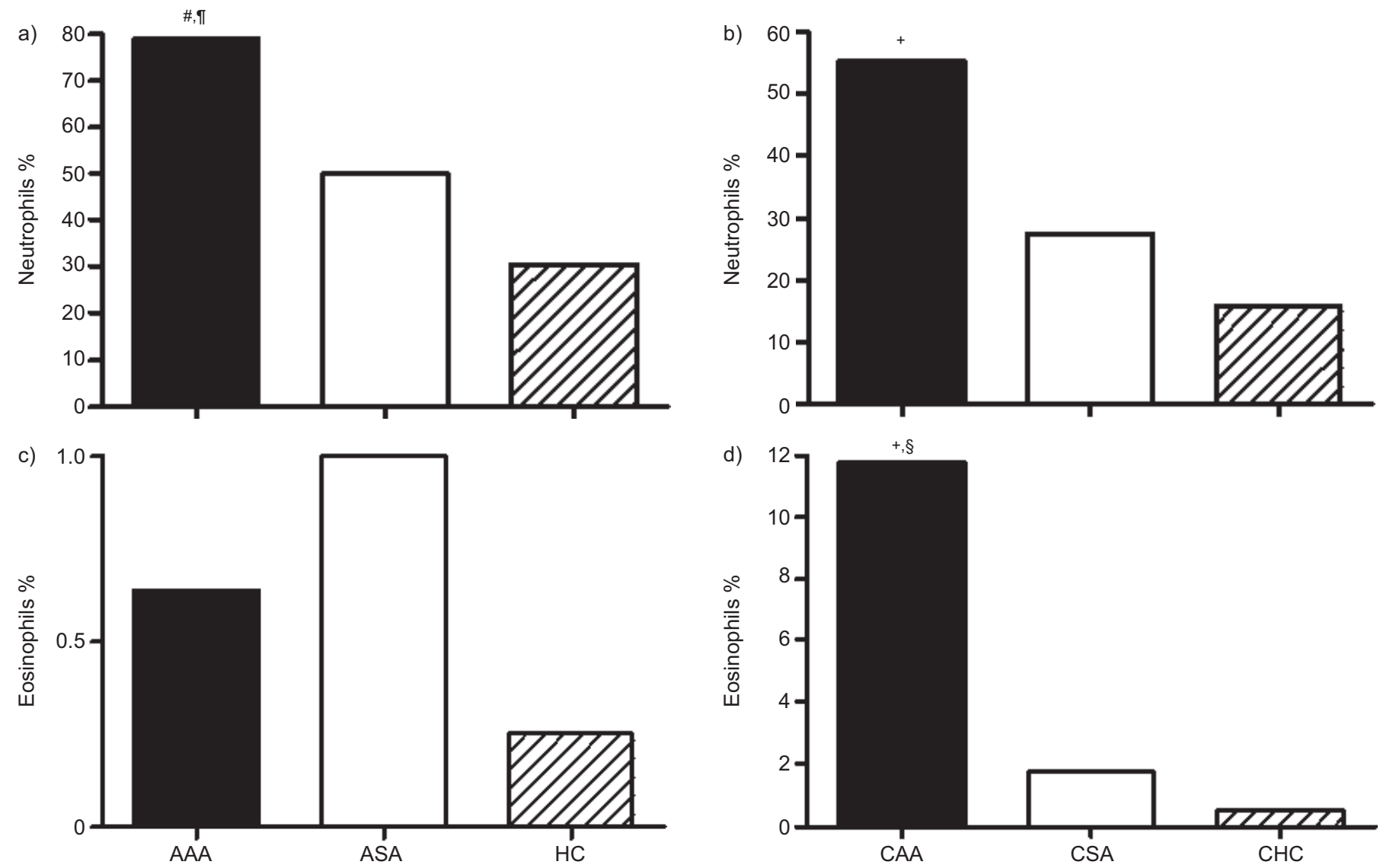

FIGURE 1. Proportion of neutrophils and eosinophils for adults and children with asthma. a) Neutrophils in adult populations, b) neutrophils in paediatric groups, c) eosinophils in adult populations and d) eosinophils in paediatric groups. AAA: adults with acute asthma, $n=22 ;$ ASA: adults with stable asthma, $n=29$; HC: healthy controls, $n=11$; CAA: children with acute asthma, $n=28$; CSA: children with stable asthma, $n=49$; CHC: child healthy controls, $n=9$. ${ }^{*}: p<0.008$ versus HCs: • : $p<0.008$ versus ASAs; ${ }^{+}: p<0.008$ versus $\mathrm{CHCs} ;{ }^{\text {s: }} \mathrm{p}<0.008$ versus CSAs.

\section{RESULTS}

\section{Demographic and clinical characteristics}

The clinical and demographic characteristics of the study subjects are shown in table 1. Compared with healthy controls, adults with asthma exhibited more atopy $(90 \%$ of ASAs and $69 \%$ of AAAs versus $18 \%$ of controls; $\mathrm{p}<0.001$ ) and significantly greater airway obstruction with lower forced expiratory volume in $1 \mathrm{~s}$ (FEV1) values than healthy controls $(71.4 \%$ and
$73.0 \%$ versus $100.8 \%$, respectively; $\mathrm{p}<0.008$ ). Subjects in the acute asthma group were slightly younger than those in the stable asthma group, but the two asthmatic groups did not differ with respect to sex or maintenance corticosteroid treatment. Children were aged between 7 and $17 \mathrm{yrs}$, and CSAs had a higher prevalence of atopy than healthy controls ( $56 \%$ versus $86 \%$; $p=0.056)$. CAAs had significantly greater airflow obstruction, with lower FEV1 \% predicted values than

\section{TABLE 3 Inflammatory phenotype by disease group for adults and children}

\begin{tabular}{|c|c|c|c|c|c|c|c|c|}
\hline & Controls & Stable asthma & Acute asthma & p-value & Controls & Stable asthma & Acute asthma & $p$-value \\
\hline Subjects & 11 & 29 & 22 & & 9 & 49 & 28 & \\
\hline \multicolumn{9}{|l|}{ Phenotype } \\
\hline Neutrophilic & $2(18.2)$ & $8(27.6)$ & $18(81.8)$ & $<0.001$ & 0 & $10(20.4)$ & $2(7.1)$ & 1.820 \\
\hline Paucigranulocytic & $9(81.8)$ & $15(51.7)$ & 0 & $<0.001$ & $9(100)$ & $24(49.0)$ & $2(7.1)$ & $<0.001$ \\
\hline
\end{tabular}


a)
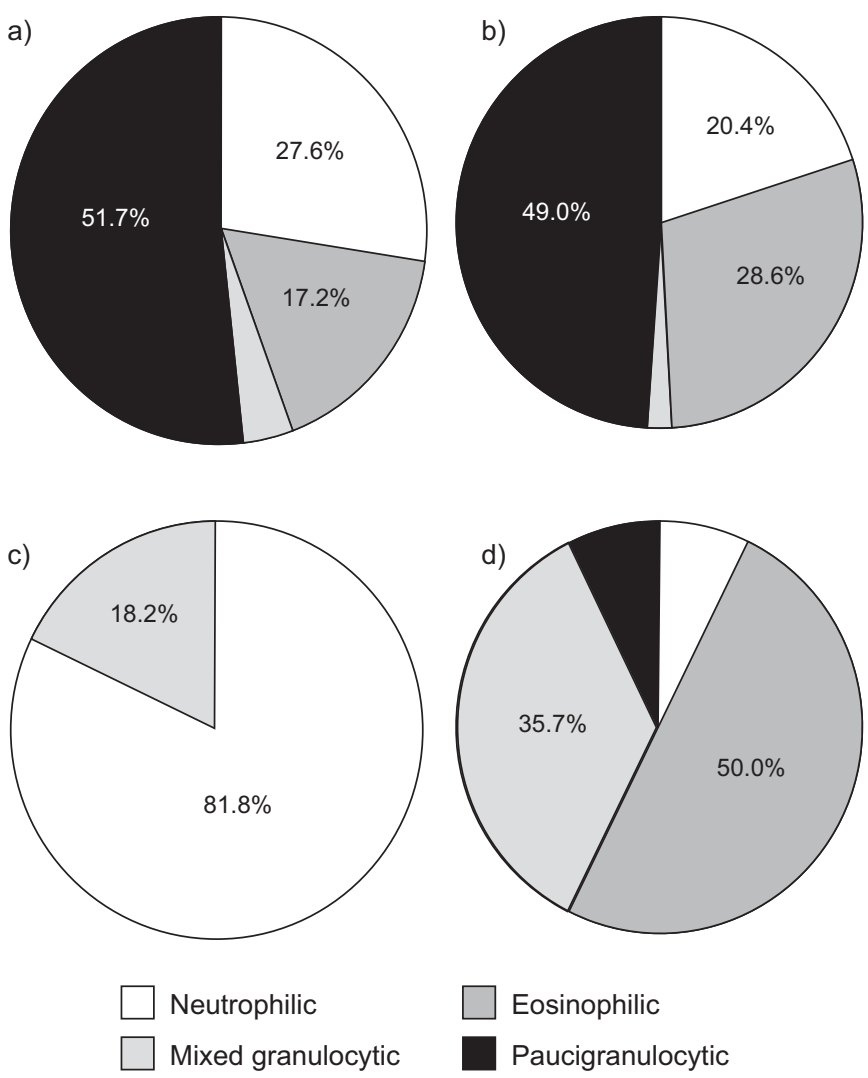

FIGURE 2. Inflammatory phenotypes in a) adults with stable asthma ( $n=29)$, b) children with stable asthma $(n=49)$, c) children with acute asthma $(n=28)$ and d) adults with acute asthma $(n=22)$.

the stable asthma group ( $53 \%$ versus $91 \% ; \mathrm{p}=0.006)$. Compared with ASAs, CSAs showed better lung function, with higher FEV1 $\%$ pred values $(p<0.001)$. However, CAAs had greater lung function impairment than the AAAs. There were fewer ex-smokers in the AAA group (18\%), compared with the ASAs $(38 \%)$ and healthy controls $(p=0.012)$. There were similar proportions of inhaled corticosteroid (ICS) users among AAAs, ASAs, CAAs and CSAs (table 1). The ICS doses were also similar, being $>1,000 \mu \mathrm{g} \cdot$ day $^{-1}$ (beclomethasone equivalent) in adults and around $480 \mu \mathrm{g} \cdot \mathrm{day}^{-1}$ in children.

\section{Analysis of inflammatory cells between healthy controls and asthmatic groups in adults and children}

The induced sputum cell quality was good in both adults and children, with similar cell viability and low levels of squamous contamination (median $<4.5 \%$ squamous cells in all groups; data not shown). Induced sputum differential cell counts are shown in table 2. The proportion of neutrophils in the AAA group was significantly higher than that in healthy adults and ASAs (fig. 1a). Sputum eosinophils were significantly higher in CAAs than in healthy children and CSA (fig. 1d). Both acute asthma groups (AAAs and CAAs) showed significantly lower percentages of macrophages compared with the healthy control and stable asthma groups; however, numbers of macrophages were not different between these groups. CAAs had lower lymphocyte counts than CSAs (table 2). Total numbers of cells did not differ between groups.
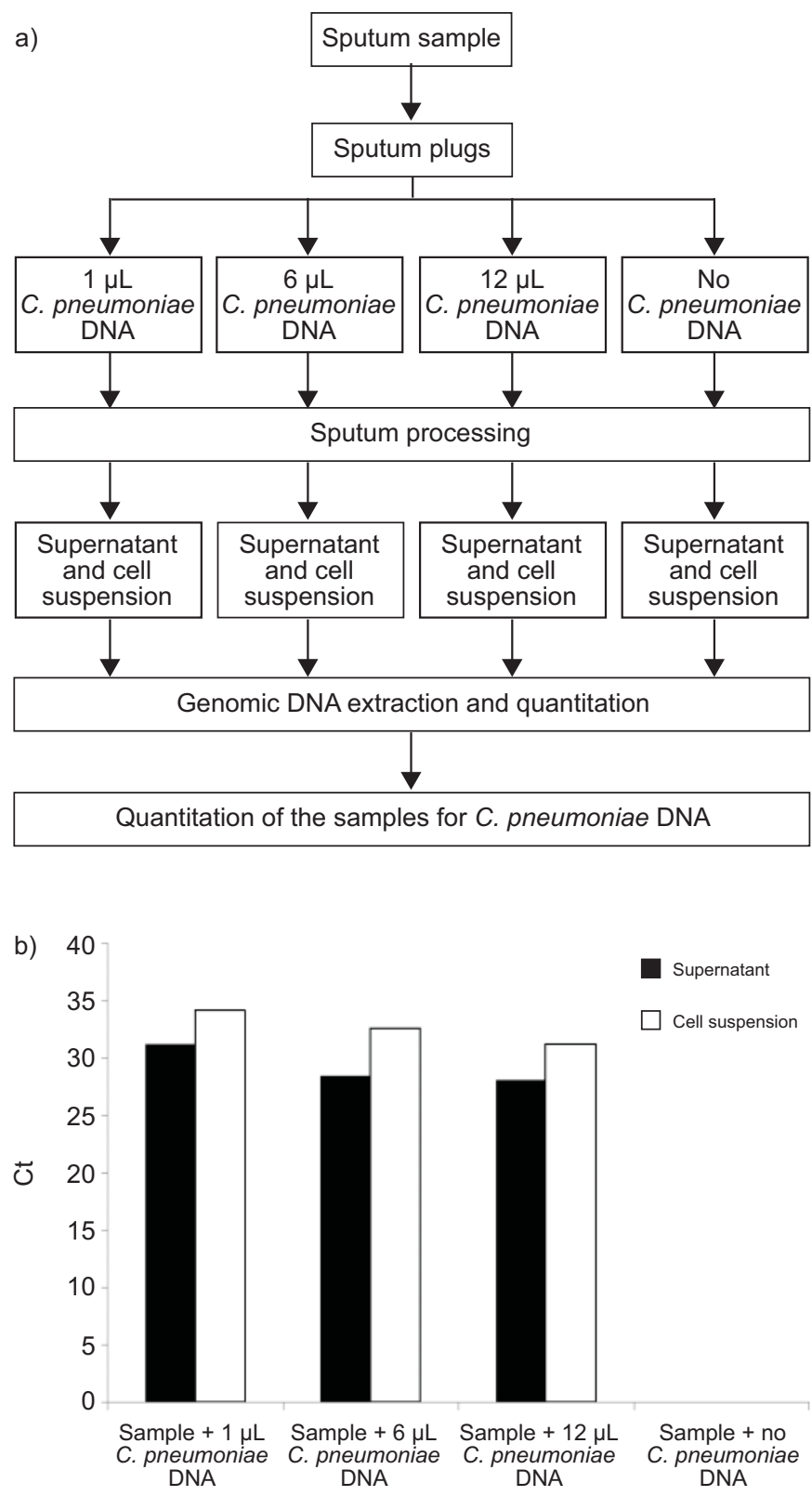

FIGURE 3. Assessment of the suitability of sample processing and purification strategies for isolation of Chlamydophila pneumoniae DNA. a) Schematic representation outlining sputum spiking with $C$. pneumoniae-positive DNA and processing. Representative sputum samples were spiked with C. pneumoniaepositive DNA and processed as described in the Materials and Methods section. b) Comparison of cycle threshold $(\mathrm{Ct})$ values of supernatant and corresponding cell suspensions upon quantitative PCR. Presence of $C$. pneumoniae-positive DNA was detected in both fractions and assumed correlation of detection threshold to input DNA can be seen.

\section{Comparison of inflammatory phenotypes between acute and stable asthma in adults and children}

All inflammatory phenotypes were observed in adults and children with stable and acute asthma, and detailed results are shown in table 3. The distribution of phenotypes was similar between ASAs and CSAs; however, there were differences in the relative frequency of the phenotypes between AAAs and 
CAAs (fig. 2). In ASAs, the most frequent inflammatory phenotype was paucigranulocytic followed by neutrophilic. Similarly, in CSA, the most frequent inflammatory phenotype was paucigranulocytic followed by eosinophilic. In AAAs, the inflammatory phenotype was predominantly neutrophilic, with the remainder being mixed granulocytic. In CAAs, the predominant phenotype was eosinophilic followed by mixed granulocytic. The pattern of inflammatory phenotype in CAAs was different to AAAs, with a significantly higher frequency of the eosinophilic subtype compared with CSAs.

\section{Real-time PCR for C. pneumoniae MOMP gene}

The differences in inflammatory phenotypes between AAAs and CAAs indicate different inflammatory processes or aetiological agents. We therefore examined C. pneumoniae infection in these subjects, hypothesising an increased prevalence in acute asthma and NA. 11 samples containing both sputum cell suspensions and sputum cell supernatants from adult healthy controls, nine samples containing sputum cell suspensions from healthy children, 26 samples containing both sputum cell suspensions and sputum cell supernatants from ASAs, 49 sputum cell supernatant samples from CSAs, 22 sputum cell supernatant samples from AAAs and 28 sputum cell supernatant samples from CAAs were tested for the presence of C. pneumoniae.

DNA was efficiently extracted from both sample types. The median DNA concentration of sputum cell supernatant was 14.2 (IQR 6.6-26.6) $\mathrm{ng} \cdot \mu \mathrm{L}^{-1}$ and there was a nonsignificant trend for more DNA to be extracted from sputum cell suspensions (25.2 (13.2-48.9) $\left.\mathrm{ng} \cdot \mu \mathrm{L}^{-1} ; \mathrm{p}=0.062\right)$. Amplification plots indicated a typical sigmoid curve for the positive control (data not shown), and flattened baseline-bound curves for the negative controls, as well as for the samples negative for $C$. pneumoniae. The sigmoid curves for $A C T B$ for each sample were within the expected cycle threshold $(\mathrm{Ct})$ range (median Ct 25.12, IQR 21.38-34.24), confirming the quality of the input DNA. In order to validate our methods, induced sputum was spiked with serial dilutions of C. pneumoniae-positive DNA, and processed and analysed for C. pneumoniae MOMP DNA by PCR. The assay was able to detect $C$. pneumoniae MOMP DNA from induced sputum with a sensitivity of $2 \times 10^{5}$ copies per $\mu \mathrm{L}$, confirming the suitability of our methodology (fig. $3 b$ ). However, using this sensitive and validated assay, $C$. pneumoniae was detected in only one sample: a CAA.

\section{DISCUSSION}

This study has identified increased airway inflammation in most cases of acute asthma, but has also shown that the inflammatory phenotypes are different between AAAs and CSAs. These results have implications for the probable mechanisms and triggers of acute asthma in adults and children, and may also impact on treatment responsiveness. We have also found that in stable asthma, the paucigranulocytic pattern was most frequent, followed by EA in children and NA in adults. Despite a high frequency of the noneosinophilic phenotypes (neutrophilic and paucigranulocytic), we were unable to confirm a role for current C. pneumoniae infection in NEA, despite using a sensitive and specific PCRbased assay. This suggests that the potential role for $C$. pneumoniae infection may be to modulate immune responses at the time of infection, which has the potential to induce a long-lasting influence on the expression of asthma.

The major finding of this study was the difference in phenotypes between AAAs and CAAs. Acute asthma is an intensely inflammatory state, since the paucigranulocytic phenotype was seen either not at all (in adults) or rarely (in children; $2 \%$ ) in this setting. The predominant inflammatory phenotype in AAAs was NA $(81.8 \%)$, whereas in CAAs it was EA. This clearly suggests that there are different inflammatory pathways involved in disease pathogenesis between AAAs and CAAs. Possible explanations for the eosinophil response seen in CAAs include allergen exposure, less maintenance corticosteroid therapy or a different response to triggers of acute asthma. CAAs did have less ICS use, but nonetheless, $68 \%$ of them were using maintenance ICS, so this is an incomplete explanation for these effects. Viral respiratory infections are the most frequent cause of acute asthma in adults and children [18]. Acute exacerbations also occur more often in children who are sensitised to aeroallergens [19]. The mixed eosinophilneutrophil responses in CAAs suggest that concurrent exposure to multiple triggers (e.g. allergens and virus infection) may be an explanation for the inflammatory response observed. This is consistent with the epidemiological observation that a combination of atopic sensitisation, allergen exposure and viral infection are required to precipitate acute asthma in children [20].

Adults exhibit an increase in IL-10 with acute asthma, which is a cytokine with known antieosinophilic effects [21]; this may explain the different responses between groups. These differences in inflammatory phenotype were accompanied by differences in clinical effects, as lung function was lower in CAAs than in AAAs. It is interesting to speculate that the treatment responses might be different between the groups. EA is typically corticosteroid sensitive, whereas the acute response to steroid in either blunted or absent in NEA [22]. These observations have been made in stable asthma, but if they also apply in acute asthma, then it would predict a lesser response to corticosteroid in AAAs and the need for other therapies active against neutrophilic responses [23].

The clinical patterns of stable asthma differ between adults and children. Adults with asthma are more likely to exhibit a persistent pattern, whereas episodic asthma is typical in children [24]. Despite these differences in clinical pattern, the inflammatory patterns were similar between adults and CSA. In our study, NEA was common in stable asthma. GIBSON et al. [25] found that a noneosinophilic pattern of inflammation was reported in $59 \%$ of 56 adults with persistent asthma. The results of our study are similar to those of RoNCHI et al. [26] who found that $28 \%$ of subjects showed increased sputum neutrophils in ASAs. The percentage was much lower in CSAs $(20.4 \%)$. However, in the present study, the prevalence of EA was higher in CSAs (30.6\%) than in ASAs (20.7\%), and this was similar to the results of CAI et al. [27]. Furthermore, the specific finding of the present study was that the proportion of subjects with paucigranulocytic asthma was greatest in ASAs (51.0\%), and a similar trend was observed in CSAs. The paucigranulocytic phenotype may represent controlled airway inflammation, which suggests it may relate to better disease prognosis. 
We also sought to examine whether C. pneumoniae infection could be detected in the NEA phenotypes. In order to do this, we used and validated a sensitive PCR-based assay and examined a wide range of clinical asthma groups. $C$. pneumoniae has been associated with adult-onset asthma [28], childhood asthma [29] and asthma severity in adults [6]. It has been postulated that NEA may represent a chronic infectious state, since many of the features mimic the responses elicited by infection, such as neutrophilia, IL-8 release and activation of TLRs [3]. Infection with C. pneumoniae has also been considered a possible cause of NEA [9]. We collected both sputum cell supernatants and suspensions of intact cells in order to maximise the chances of detecting both intracellular and extracellular C. pneumoniae [11]. We included appropriate controls in the assay, and the results of positive controls and the $A C T B$ control demonstrate that the methods we used were effective. The detection results were mostly negative in the different clinical populations of the present study, except one sample from a CAA. We interpret our results to indicate that current active acute or chronic infection is uncommon in our population. This stands in contrast to other populations, particularly in Europe, where C. pneumoniae infection is more prevalent. Based on studies of C. pneumoniae infection in neonates, we cannot exclude the possibility that immunodeviation following resolved C. pneumoniae infection in early life may be a mechanism that modulates NEA.

Potential limitations of the present study relate to the sample size and the selection methods for subjects. There was good power for the analyses involving NA in adults and children, and EA in children (acute and stable); however, the small sample size meant limited power to assess the eosinophilic phenotype in AAAs (power 40\%). Similarly, using subjects selected from a database may introduce selection bias. However, the same data collection methods were used for all participants, where a staff member attended the emergency department to recruit adults and children with acute asthma. As the adult and paediatric emergency rooms are co-located, there was no bias that could arise from differential recruitment. The main limitation to this method was the amount of time that the staff member could spend in the emergency room (i.e. during working hours). The different FEV1 results between AAAs and CAAs may reflect timing of measurement after $\beta$-agonist dosing. While all subjects had FEV1 measured after $\beta$-agonist pre-treatment, the number of additional doses before induction was not controlled and may have affected comparisons between groups.

In summary, the present findings provide evidence for differences in the prevalence of inflammatory phenotypes in AAAs and CAAs. These differences were associated with clinically significant alterations in lung function and suggest the involvement of different mechanistic pathways in acute asthma in children and adults. CAAs showed high eosinophilic inflammatory responses, whereas neutrophilic responses were most frequent in AAAs. The paucigranulocytic phenotype was the major pattern observed in both ASAs and CSAs, although heterogeneity in inflammatory phenotypes was a common feature in stable asthma. Current C. pneumoniae infection does not appear to be a common reason to explain the NEA phenotype in asthma, or to increase respiratory acute exacerbation.

\section{STATEMENT OF INTEREST}

A statement of interest for P.G. Gibson can be found at www.erj. ersjournals.com/site/misc/statements.xhtml

\section{ACKNOWLEDGEMENTS}

We thank H. Powell for her statistical assistance and N. Fibbens for data collection (both John Hunter Hospital, Newcastle, NSW, Australia).

\section{REFERENCES}

1 Ten Brinke A, Zwinderman AH, Sterk PJ, et al. Factors associated with persistent airflow limitation in severe asthma. Am J Respir Crit Care Med 2001; 164: 744-748.

2 Simpson JL, Scott R, Boyle MJ, et al. Inflammatory subtypes in asthma: assessment and identification using induced sputum. Respirology 2006; 11: 54-61.

3 Simpson JL, Grissell TV, Douwes J, et al. Innate immune activation in neutrophilic asthma and bronchiectasis. Thorax 2007; 62: 211-218.

4 Horvat JC, Starkey MR, Kim RY, et al. Chlamydial respiratory infection during allergen sensitization drives neutrophilic allergic airways disease. J Immunol 2010; 184: 4159-4169.

5 Droemann D, Rupp J, Goldmann T, et al. Disparate innate immune responses to persistent and acute Chlamydia pneumoniae infection in chronic obstructive pulmonary disease. Am J Respir Crit Care Med 2007; 175: 791-797.

6 Black PN, Scicchitano R, Jenkins CR, et al. Serological evidence of infection with Chlamydia pneumoniae is related to the severity of asthma. Eur Respir J 2000; 15: 254-259.

7 Lieberman D, Lieberman D, Printz S, et al. Atypical pathogen infection in adults with acute exacerbation of bronchial asthma. Am J Respir Crit Care Med 2003; 167: 406-410.

8 Daian CM, Wolff AH, Bielory L. The role of atypical organisms in asthma. Allergy Asthma Proc 2000; 21: 107-111.

9 Simpson JL, Powell H, Boyle MJ, et al. Clarithromycin targets neutrophilic airway inflammation in refractory asthma. Am J Respir Crit Care Med 2008; 177: 148-155.

10 Horvat JC, Starkey MR, Kim RY, et al. Early-life chlamydial lung infection enhances allergic airways disease through age-dependent differences in immunopathology. J Allergy Clin Immunol 2010; 125: 617-625.

11 Harju TH, Leinonen M, Nokso-Koivisto J, et al. Pathogenic bacteria and viruses in induced sputum or pharyngeal secretions of adults with stable asthma. Thorax 2006; 61: 579-584.

12 Kuoppa Y, Boman J, Scott L, et al. Quantitative detection of respiratory Chlamydia pneumoniae infection by real-time PCR. J Clin Microbiol 2002; 40: 2273-2274.

13 Apfalter P, Barousch W, Nehr M, et al. Comparison of a new quantitative ompA-based real-time PCR TaqMan assay for detection of Chlamydia pneumoniae DNA in respiratory specimens with four conventional PCR assays. J Clin Microbiol 2003; 41: 592-600.

14 Wark PA, Johnston SL, Simpson JL, et al. Chlamydia pneumoniae immunoglobulin A reactivation and airway inflammation in acute asthma. Eur Respir J 2002; 20: 834-840.

15 Norzila MZ, Fakes K, Henry RL, et al. Interleukin-8 secretion and neutrophil recruitment accompanies induced sputum eosinophil activation in children with acute asthma. Am J Respir Crit Care Med 2000; 161: 769-774.

16 Simpson JL, McElduff P, Gibson PG. Assessment and reproducibility of non-eosinophilic asthma using induced sputum. Respiration 2010; 79: 147-151.

17 Pin I, Gibson PG, Kolendowicz R, et al. Use of induced sputum cell counts to investigate airway inflammation in asthma. Thorax 1992; 47: 25-29. 
18 Jackson DJ, Johnston SL. The role of viruses in acute exacerbations of asthma. J Allergy Clin Immunol 2010; 125: 1178-1187.

19 Sly PD, Boner AL, Björksten B, et al. Early identification of atopy in the prediction of persistent asthma in children. Lancet 2008; 372: 1100-1106.

20 Green RM, Custovic A, Sanderson G, et al. Synergism between allergens and viruses and risk of hospital admission with asthma: case-control study. BMI 2002; 324: 763.

21 Grissell TV, Powell H, Shafren DR, et al. Interleukin-10 gene expression in acute virus-induced asthma. Am J Respir Crit Care Med 2005; 172: 433-439.

22 Green RH, Brightling CE, Woltmann G, et al. Analysis of induced sputum in adults with asthma: identification of subgroup with isolated sputum neutrophilia and poor response to inhaled corticosteroids. Thorax 2002; 57: 875-879.

23 Johnston SL, Blasi F, Black PN, et al. The effect of telithromycin in acute exacerbations of asthma. $N$ Engl J Med 2006; 354: $1589-1600$
24 Gelfand EW, M Kraft. The importance and features of the distal airways in children and adults. J Allergy Clin Immunol 2009; 124: Suppl. 6, S84-S87.

25 Gibson PG, Simpson JL, Saltos N. Heterogeneity of airway inflammation in persistent asthma: evidence of neutrophilic inflammation and increased sputum interleukin-8. Chest 2001; 119: 1329-1336.

26 Ronchi MC, Piragino C, Rosi E, et al. Role of sputum differential cell count in detecting airway inflammation in patients with chronic bronchial asthma or COPD. Thorax 1996; 51: 1000-1004.

27 Cai Y, Carty K, Henry RL, et al. Persistence of sputum eosinophilia in children with controlled asthma when compared with healthy children. Eur Respir J 1998; 11: 848-853.

28 Hahn DL, Dodge RW, Golubjatnikov R. Association of Chlamydia pneumoniae (strain TWAR) infection with wheezing, asthmatic bronchitis, and adult-onset asthma. JAMA 1991; 266: 225-230.

29 Cunningham AF, Johnston SL, Julious SA, et al. Chronic Chlamydia pneumoniae infection and asthma exacerbations in children. Eur Respir J 1998; 11: 345-349. 